\title{
The Epidemiological Situation of Avian Salmonellosis in the Republic of Moldova
}

\author{
Starciuc Nicolae*, Juncu Olga and Osadci Natalia \\ Faculty of Veterinary Medicine, Department Clinics II, Moldova, Europe
}

Received: 㭗: December 10, 2018; Published: 制: December 18, 2018

*Corresponding author: Starciuc Nicolae, Faculty of Veterinary Medicine, Department Clinics II, Moldova, Europe

\begin{abstract}
The goal of the proposed research was to establish the diversity of the pathogenic serotypes of Salmonella spp. in the poultry enterprises which produce meat and eggs for consumption and to determine the critical points of carcasses and eggs contamination which could lead to risks of toxic infections in humans. The samples for microbiological investigations were collected from the poultry carcasses and egg shells delivered to the Central Agricultural Market of Chisinau from different poultry companies in the country, including equipment, vehicles for poultry transportation and manure. The inseminations were performed on nutrient media like: bismuth sulfite agar and Salmonella Sighela Agar. The bacteriological investigations demonstrated that the majority of the examined samples showed the presence of colonies of Salmonella spp. The results demonstrated that in the samples taken from poultry products (carcasses, meat products and eggs) Salmonella spp. serotypes as S. Infantis, S. enteritidis, S. typhimurium have prevailed; however, in the samples taken from poultry equipment and vehicles, prevailed the serotypes as $S$. enteritidis, S. Derby, S. gallinarum and $S$. Infantes. The data gathered confirmed that some pathogenic serotype of Salmonella spp., in particular S. enteritidis and S. typhimurium persist in the poultry products and can be a source of risk of birds salmonellosis and human toxic infections.
\end{abstract}

Keywords: Carcasses; Lavages; Contamination; Microorganisms; Microbial Colonies

\section{Introduction}

It is known that poultry meat is often contaminated with pathogens that can be dangerous for humans. At present, the microorganisms of the genus Salmonella spp. and Campylobacter are the most common hazards associated with infected poultry consumption $[1,4,6]$. From approximately $20-25 \%$ of all cases of salmonellosis, it is estimated that Salmonella spp. illness appears consecutively of infected poultry consumption [3]. Another important source of toxic infections in humans is poultry eggs which can be contaminated with Salmonella spp. During the sale, as well as in contact with the external environment (air, equipment, packaging, staff, transport, etc.), the most frequently pathogenic serotypes of Salmonella spp. is S. enteridis the S. galinarium [2,5,7].

Taking into consideration the role of domestic and wild birds and their products (meat and eggs) in studying the incidence of bird's salmonellosis and food toxic infections in humans; therefore, the goal of our research was focused on establishing the spread of avian salmonellosis on the territory of the republic as well as determining the isolation methods of pathogenic serotypes of Salmonella spp.

\section{Materials and Methods}

As research material served meat samples taken from poultry carcasses sold in the commercial units of the Central Agricultural Market of Chisinau, delivered from different poultry farms in the republic. In total there were 45 samples collected and examined. Simultaneously, the egg samples for investigation (45 samples) were collected from the units specialized in the marketing of eggs in the Central Agricultural Market of Chisinau, delivered from poultry enterprises producing eggs. Also, were collected 35 samples of material for investigation from the poultry equipment, vehicles and faces. The inseminations were carried out using culture media as: bismuth sulfite agar (BSA), and Salmonella Sighiella Agar (SSA). Some investigations (serotyping) were performed at the Republican Veterinary Diagnostic Center.

\section{Results/Observations}

The monitoring of the Salmonella spp. colonies presence and their morphological structure was studied on the surface of the sample (poultry carcasses) as well as their inside samples. Some of 
the results of this study are shown in Figures $1 \& 2$. In cases when the inseminations were performed from the surface of the carcasses on bismuth sulfite agar (Figure1) on all samples was established a massive growth of Salmonella spp. colonies, placed on Petri dish in form of chain, separate or in piles, their number having variations within 66 to 168 colonies. When the inseminations were performed from the inside of the samples the colonies number had variations within 12 to 57 colonies.

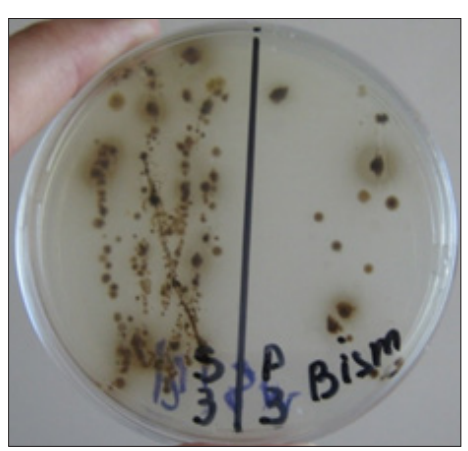

Figure 1: Colonies of Salmonella spp. (medium BSA), insemination from the surface and depth of the sample.

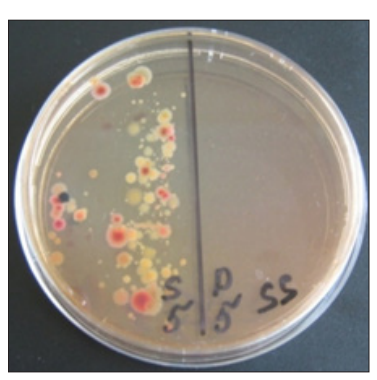

Figure 2: Colonies of Salmonella spp. (medium SSA), insemination from the surface and inside of the sample.

On the SSA (Figure 2), the number of Salmonella colonies varied within 89 to 166 colonies on the surface of the samples and from 0 to 42 colonies, from the inside of the samples. An intensive growth of the colonies was established in cases when the inseminations were performed from the lavages collected from the surface of the egg shells (Figure 3) having values within 22 to145 colonies on bismuth sulfite agar. When the inseminations were performed from the surfaces of the spaces, the colony number was lower and ranged from 12 to 110 colonies.

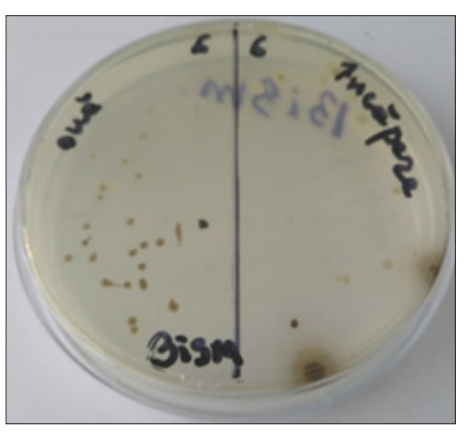

Figure 3: Colonies of Salmonella spp. (lavages surface of the eggs and poultry building), on BSA.

An intensive growth of Salmonella spp. colonies was established also when the insemination was performed from the lavages taken from the walls of transport units and cells for bird's transportation (Figure 4). For the lavages taken from inside of the vehicles the number of the colonies had values within the limits from 78 to 95 colonies, while those taken from the cells for bird's transportation and spaces for their maintenance on the market had values within the limits of 87 to 215 colonies. Some microbiological investigations (serptipisation) were performed in collaboration with the department of microbiology of Republican Veterinary Diagnostic Center. In the Table 1 are presented the data on positive samples taken from poultry carcasses which were confirmed positive for Salmonella spp.

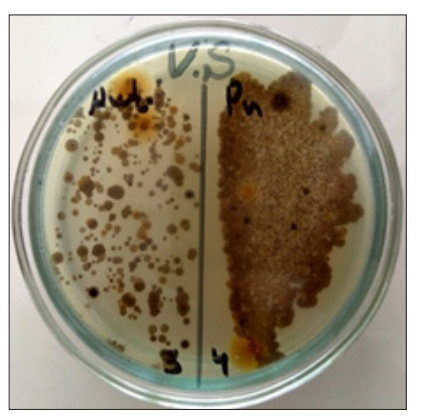

Figure 4: Colonies of Salmonella spp. (the lavages from the vehicles and birds' cells), on BSA.

Table 1: Serotypes of Salmonella spp. isolated from the poultry products.

\begin{tabular}{|c|c|c|c|}
\hline Nr. d/o & Serotype of Salmonella spp. & Name of the product & Nr. of positive sample \\
\hline 1 & Salmonella Enteritidis & Poultry meat & 4 \\
\hline 2 & Salmonella Infantis & Poultry meat & 10 \\
\hline 3 & Salmonella Winneba & Poultry meat & 3 \\
\hline 4 & Salmonella Newport & Poultry meat & 2 \\
\hline 5 & Salmonella Uppsala & Poultry meat & 1 \\
\hline 6 & Salmonella Fillmore & Poultry meat & 1 \\
\hline 7 & Salmonella Typhimurium & Mechanically deboned poultry meat & 2 \\
\hline 8 & Salmonella Dessau & Poultry meat & 1 \\
\hline 9 & Salmonella Farsta & Mechanically deboned poultry meat & 5 \\
\hline
\end{tabular}

Cite this article: Starciuc N, Juncu O, Osadci N. The Epidemiological Situation of Avian Salmonellosis in the Republic of Moldova. Biomed J Sci 
The results from Table 1 shows that during 2018 in the Republican Veterinary Diagnostic Center, 30 samples of poultry products were confirmed with positive Salmonella spp. including serotypes as $S$. enteritidis and $S$. tiphimurium which present a real danger for public health. The serotypes $S$. infantis showed the highest rate of contamination in poultry carcasses being present in 15 samples; therefore, representing $50 \%$ of the total number of salmonella serotypes.

In addition, positive samples with Salmonella spp. were confirmed in the following poultry products presented in Table 2. The results of the bacteriological investigations (Table 2) show the presence and circulation of the pathogenic serotypes of Salmonella spp., in particular S. enteritidis, S. gallinarum, in poultry products as well as in buildings for chipping chickens.

Table 2: Monitoring of serotypes of Salmonella spp. in some objects and poultry products.

\begin{tabular}{|c|c|c|c|}
\hline Nr. d/o & Type of the sample & Number of samples & Serotype of Salmonella spp. \\
\hline 1 & Eggs & 3 & S. enteritidis \\
\hline 2 & Pelimene & 3 & S. derby \\
\hline 3 & Pelimene & 4 & S. infantis \\
\hline 4 & Pelimene & 2 & S. enteritidis \\
\hline 5 & Meat products & 5 & S. enteritidis. \\
\hline 6 & Chickens 6 day old & 5 & S. gallinarum \\
\hline 7 & Chickens 12 day old & 5 & S. gallinarum \\
\hline 8 & Quail chickens & 5 & S. galinarum \\
\hline 9 & Shoe (from chickens building, 24 day old) & 10 & S. enteritidis \\
\hline 10 & Shoe (from chickens building, 36 day old) & 2 & S. enteritidis \\
\hline
\end{tabular}

\section{Conclusion}

a) The epidemiological and microbiological investigations confirmed that some pathogenic serotype of Salmonella spp., especially $S$. Enteritidis and S. typhimurium persist in the poultry flocks and in poultry products which present a risk of birds and human contamination, thus giving a reason for further multilateral and thorough study of this disease, including the public health sector.

b) Serotyping cultures of Salmonella spp. showed that in the samples of poultry products (meat, meat products and eggs) have prevailed serotype $S$. Infantis, S. enteritidis, S. typhimurium and $S$. winneba, while in the samples of poultry flocks and buildings, serotypes $S$. enteritidis, $S$. derby, $S$. gallinarum and $S$. Infantes were more present.

\section{Discussion}

The obtained results have shown that currently the problem of poultry salmonellosis is existing in the republic. Isolation of pathogenic serotype of Salmonella spp. from the poultry carcasses, eggs, equipment, from birds' feces, indicated the existing risk of contamination of the poultry at some stages of production, processing, or marketing with pathogenic serotypes of Salmonella spp., which could eventually develop salmonellosis in birds and humans. Taking into consideration this situation it is important to continue research on this thematic multilaterally. Simultaneously is necessary to analyze if the situation requires the possible use of some vaccine strains, that consequently will allow to study their effectiveness and impact on epidemiological situation and public health.

\section{References}

1. Amélie Rouger, Odile Tresse, Monique Zagorec (2017) Bacterial Contaminants of Poultry Meat: Sources, Species and Dynamics. J Microorganisms 5(3): 50.

2. Andiara Gonçalves-Tenório, Beatriz Nunes Silva, Vânia Rodrigues, Vasco Cadavez, Ursula Gonzales-Barron (2018) Prevalence of Pathogens in Poultry Meat: A Meta-Analysis of European Published Surveys. J Foods 7(5): 69.

3. Arnold ME, Martelli F, McLaren I, Davies RH (2014) Estimation of the rate of egg contamination from Salmonella-infected chickens. Zoonosis Public Health 61(1): 18-27.

4. Durul B, Acar S, Bulut E, Kyere EO, Soyer Y (2015) Subtyping of Salmonella Food Isolates Suggests the Geographic Clustering of Serotype Telaviv. Foodborne Pathog Dis 12(12): 958-965.

5. Gulay Firildak, Ahmet Asan, Erman Goren (2015) Chicken Carcasses Bacterial Concentration at Poultry Slaughtering Facilities. Asian Journal of Biological Sciences 8(1): 16-29.

6. Kottwitz LB, Leão JA, Back A, Rodrigues Ddos P, Magnani M, et al. (2013) Commercially laid eggs vs. discarded hatching eggs: Contamination by Salmonella spp. Braz J Microbiol 44(2): 367-370.

7. Mouttotou N, Ahmad S, Kamran Z, Koutoulis KC (2017) Prevalence, Risks and Antibiotic Resistance of Salmonella in Poultry Production Chain. In Tech; Rijeka, Croatia pp. 215-234. 


\section{ISSN: 2574-1241}

DOI: 10.26717/BJSTR.2018.12.002234

Starciuc Nicolae. Biomed J Sci \& Tech Res

(c) (i) This work is licensed under Creative

Submission Link: https://biomedres.us/submit-manuscript.php

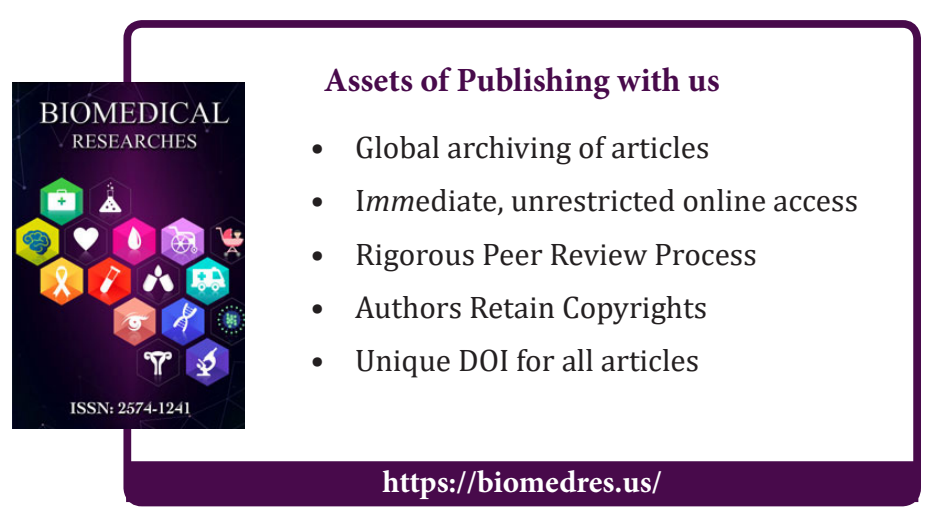

